\title{
Costos directos de producción en la rentabilidad del cultivo de la papaya en la provincia de Mariscal Cáceres
}

\author{
Martha Liz Reátegui Reátegui \\ mlreategui@unsm.edu.pe \\ Rosa Elena Cueto Orbe \\ recuetoo@unsm.edu.pe \\ Sandra Ruiz Correa \\ sruiz_correa@hotmail.com
}

Lady Diana Arévalo Alva

larevaloa@unsm.edu.pe.com

Blanca Yalta Flores

byaltf@unsm.edu.pe

Luis Alberto Ríos López

lrios@unsm.edu.pe.com

Harry Saavedra Alva

hsaavedraa@unsm.edu.pe

Escuela de post grado

\section{RESUMEN}

Universidad César Vallejo

La investigación se centra principalmente en establecer el dominio de los costos directos de producción y la rentabilidad del cultivo de la papaya en la Provincia de Mariscal Cáceres. Se justifica, por que nos servirá para contrastar la praxis con la teoría desarrollada sobre la influencia de productos agrarios basados en los costos de la producción. El objetivo es determinar la influencia de los costos directos de producción y la rentabilidad del cultivo de la papaya en la Provincia de Mariscal Cáceres. Se apeló al tipo de estudio aplicativo desarrollado en el nivel descriptivo correlacional, diseño transversal. Como técnicas de recolección de datos se ha establecido la observación, sobre los costos de la producción en una hectárea del cultivo de la papaya. La encuesta, para recoger información de los 50 productores. Las entrevistas, desarrolladas de modo informal a productores. Se concluye que, se logró identificar los costos directos e indirectos de la producción del cultivo de la papaya como es en el año 1 instalación y en el año 2 mantenimiento.

Palabras clave: costos; producción; rentabilidad; tecnología. 


\title{
Direct production costs in the profitability of papaya cultivation in the province of Mariscal Cáceres
}

\begin{abstract}
The research is mainly focused on establishing the domain of direct production costs and profitability of papaya cultivation in the Province of Mariscal Cáceres. It is justified, because it will help us to contrast the praxis with the theory developed on the influence of agricultural products based on the costs of production. The objective is to determine the influence of direct production costs and profitability of papaya cultivation in the Province of Mariscal Cáceres. The type of applicative study developed at the correlational descriptive level, cross-sectional design, was used. As data collection techniques, observation has been established on the production costs in one hectare of papaya cultivation. The survey, to collect information from the 50 producers. The interviews, developed informally with producers. It is concluded that, it was possible to identify the direct and indirect costs of the production of the papaya crop as it is in year 1 installation and in year 2 maintenance.
\end{abstract}

Keywords: costs; production; cost effectiveness; technology.

Artículo recibido: 05 octubre. 2021 Aceptado para publicación: 02 noviembre 2021 Correspondencia: mlreategui@unsm.edu.pe Conflictos de Interés: Ninguna que declarar 


\section{INTRODUCCIÓN}

En la actualidad población de la región San Martín se sustenta básicamente de la actividad agrícola y uno de los principales productos que se cultiva en la ciudad de Juanjuí, en estos últimos años es la papaya. Según el Ministerio de Agricultura, la papaya representa el $20.0 \%$ de la producción total de la Provincia de Mariscal Cáceres, tal es así que en el año 2007 se sembró 50 Ha de papaya y en el 2008200 Ha a partir del mes de septiembre, logrando una producción de 15-20TN/ Ha.

En el cultivo de la papaya, la falta de desconocimiento de los agricultores y la inexperiencia de ellos por personas que venían de otros departamentos en busca de tierras para este sembrío, y en plazo corto para la cosecha logrando rentabilidad en sus ingresos económicos y emocionándose por el precio que ofrecía el mercado hicieron que se dediquen a un $100 \%$ a este cultivo dejando de lado a los productos alternativo que tenían conocimiento dentro de la zona.

Es por ello que el desconocimiento de este cultivo dentro de la Provincia de Mariscal Cáceres por el sembrío de Hectáreas de papaya se propago en toda la zona no contando en la ciudad de Juanjuí con ningún registro ni control de producción de este cultivo por parte del Estado y del agricultor, desconociéndose la rentabilidad real de este cultivo por lo que se necesita conocer y explicar los costos directos de la producción; cuanto se gana o pierde el agricultor al dedicarse este cultivo de la papaya.

\section{Actividad Agrícola}

La actividad agrícola es un conjunto de operaciones necesarias para convertir los suelos en productivos, con el objeto de obtener productos vegetales (Manjarrés, 2003), "sin causar daño al ecosistema de la zona o del área específica donde desarrollemos la actividad" (Clavijo, P. 1998, p.23).

Según Clavijo (1998) el objetivo de toda explotación agrícola es lograr el máximo rendimiento de las tierras y la mejora de la calidad de los productos, lo cual requiere del uso adecuado de paquetes tecnológicos, como la mecanización de las tareas agrícolas, el uso más racional de los fertilizantes y los agroquímicos, el uso de nuevas variedades y la selección de buenas semillas, el empleo racional y ecológico de los sistemas de control y represión de las plagas, la administración eficiente de los recursos físicos, humanos y técnicos, enfocada hacia el mayor rendimiento económico de la explotación, en beneficio de un mejor nivel de vida de los agricultores y su familia en el desarrollo del país. 
El cultivo de la papaya se adapta en los límites de los 32 a 35 grados de latitud norte y de 32 a 35 grados de latitud sur, en las zonas tropicales y subtropicales. Lo que corresponde a áreas cálidas que están comprendidas desde el nivel del mar hasta los 1000 metros; pero los mejores rendimientos y calidad de frutos se obtienen entre los 0-600 m.s.n.m. (sag.gob.hn, 2005). A medida que la papaya se produce a mayor altura se desarrollan fruto insípido debido una menor capacidad de conversión de azúcares. El óptimo de temperatura oscila entre los 25 a $380 \mathrm{C}$ y la humedad relativa de 60 a $85 \%$. La planta requiere de una buena distribución de las lluvias, entre 1,500 a 2,000 mm de precipitación durante el año para su normal desarrollo (Adriazola, 1999).

Debido al alto contenido de agua en los frutos y a la constante formación de estos el cultivo requiere de agua durante todo el año para asegura una cosecha sin interrupciones. Además, la planta requiere de alta luminosidad para que los frutos alcancen un contenido de azúcares deseable por lo que no se debe intercalar con otros cultivos que pudieran darle sombra.

El papayo se desarrolla muy bien en suelos de textura franco, No son recomendables del tipo franco-arenoso por el riesgo de ataque de nematodos (IIAP, 2000) aunque se puede cultivar en cualquier otro tipo de suelo siempre y cuando tenga una profundidad mínima de $0.40 \mathrm{~m}$., buena capacidad de retención de agua, así como facilidad para eliminar el exceso de esta. Para el drenaje externo son convenientes los terrenos con leves pendientes; las raíces de papayo son muy susceptibles a morir por falta de oxígeno de ahí la importancia del drenaje. Además, es favorable que el pH del suelo oscile entre 5.5 y 7.5 y que tenga un buen contenido de materia orgánica.

Las semillas sirven para eliminar parásitos y las hojas como antimalárico y antiasmático, mientras que sus enzimas curan heridas y enfermedades oculares (Elcomercioperu.com, 2004).

Su látex, el líquido transparente similar a la goma, contiene la enzima papaína que ayuda a digerir las proteínas y es buenísima para el hígado. Esto se debe en gran medida a su composición química rica en vitamina A, C y B que sumados a la carpaína, alcaloide especializado en trabajar sobre el líquido biliar, la proveen de los elementos indispensables para digerir carnes y comidas pesadas (dsalud.com2004). Sin embargo, Abeles, citado por Alvarado (1995), manifiesta que todas las partes de la planta contiene un látex, pero que se extrae principalmente del fruto verde. A medida que el fruto madura 
su contenido de látex disminuye y al momento de consumo prácticamente no contiene nada. Además de presentar estas importantes cualidades nutritivas, ayuda también a la quema de grasas con lo que desarrolla un efecto adelgazante, desintoxicante y depurativo en forma natural. En terapias digestivas se la utiliza para compensar la falta de secreciones gastroduodenales y pancreáticas; su acción suavizante y antiséptica sobre las mucosas digestivas la hacen muy útil en el caso de gastroenteritis y colitis de cualquier tipo, así como en situaciones de estreñimiento o colon irritable. Dsalud.com, (2004).

Los Factores que afectan la Influencia de la producción de papaya, dentro del proceso de cultivo y cosecha del fruto pueden surgir una diversidad de factores que alteren la calidad y condiciones generales del producto, como daños físicos y mecánicos, plagas, mala administración de funguicidas, problemas de empaque y maniobrabilidad del producto, condiciones inadecuadas de transporte entre otras. En el proceso de cultivo la presencia de plagas y enfermedades es otro problema a considerar. En su primera edad puede regarse normalmente al campo, pero cuando las plantas alcanzan $1.0 \mathrm{~m}$ de estatura los riegos deben restringirse gradualmente. El exceso de agua favorece el ataque de Phytopthora sp., Phytium aphani dermatum, Rhizoctonia sp., Clamidos porium sp., entre otras, y además causa asfixia en las plantas pudiendo matarlas si no se controla adecuadamente (Calzada, 1983: 102).

En las épocas frías y húmedas las bayas y frutos son atacados por oldium (oidum caricae). La virosis hace daño en la selva y es ocasionada por tres virus: Mosaico, Punta anucimada o Bunchy Top y Hoja distorsionada (Calzada, 1983:102).

En cuanto a plagas el papayo es atacado por la Arañita Roja (Te tranychus sp.) y pulgones (Alphis sp.); a las raíces es atacada por el nematodo (Heterodera marioni) especialmente si se cultiva en suelos arenosos, previniéndose este plaga escogiendo terrenos ricos en materia orgánica y haciendo un buen abonamiento. La fruta de papaya mientras está en la planta y después de cosechada es atacada por varios hongos tales como Rhizopus sp., Penecillium sp., Geotrichum sp., Collatotrichum sp., Phytopthora sp., Cladosporium sp., Fusarium sp., entre otros. Estos hongos afectan la producción en mayor o menor grado ocasionando pérdidas que en promedio llegan al 25\% de la fruta producida (Calzada, 1983: 102).

\section{Ciclo de Producción Agrícola}


El ciclo de producción de una empresa agrícola comprende una serie de fases que se inician con la preparación de los suelos y finalizan con la cosecha del cultivo y la comercialización del producto. El éxito del proceso productivo depende del cuidado que se le dé a cada una de estas etapas, además, a través de las mismas se puede realizar el análisis de los costos.

En cuanto al ciclo de producción de la papaya (Monasterio, M. 1980) citado por Manjarrés (2003), señala que éste depende de la altitud, a partir de los 600 hasta los 1000 metros sobre el nivel del mar, es decir, entre la Provincia de Mariscal Cáceres, Huallaga y Bellavista del Departamento San Martín se cultiva la papaya Criolla, la misma tiene un buen desarrollo en las zonas altas, cuyo ciclo productivo es largo (240 días), lo que permite obtener una cosecha al 8vo mes de la plantación.

En cada una de estas etapas se incurre en costos de materia prima e insumos (semilla, fertilizantes, abonos, fungicidas, herbicidas e insecticidas) que se transforman en producto terminado: papaya para consumo o papaya para semilla, con la adhesión de mano de obra directa (jornales) y otros costos indirectos. etc.

\section{Sistemas de Costos}

Son el conjunto de procedimientos, técnicas, registros e informes estructurados sobre la base de la teoría de la partida doble y otros principios técnicos, que tienen por objeto la determinación de los costos unitarios de producción y el control de las operaciones fabriles.

Se busca contribuir a la planeación de utilidades proporcionando anticipadamente los costos de producción, distribución, administración y financiamiento. Contribuir en la elaboración de los presupuestos de la empresa, programas de producción, ventas y financiamiento.

Sistema de costeo. Un sistema de costeo implica un conjunto de procedimientos, técnicas, registros e informes estructurados que tienen por objeto la determinación del costo de producción y el control de las operaciones de fabricación efectuadas. De acuerdo con las características del proceso estos sistemas d acumulación se clasifican en: sistema de órdenes de producción y sistema de costos por proceso.

\section{Procedimientos de control.}

Por órdenes de producción. Para el control de cada partida de artículos se requiere, por consiguiente, la emisión de una orden de producción. En este procedimiento cada persona 
produce a través de órdenes y generalmente se hace por lotes, lo que hace que la identificación de costos sea más específica.

Por procesos productivos. Se presenta cuando la producción no está sujeta a interrupciones, sino que se desarrolla en forma continua e interrupción, de tal manera que no es posible tomar decisiones aisladas para producir uno u otro artículo, sino que la producción está sujeta a una secuencia durante periodos indefinidos. La producción es en serie o en línea. En el Sistema de costos por procesos, el costo se obtiene hasta que se hace el cierre o inventario final, y siempre se calcula de manera más general.

\section{Costos de Producción Agrícola}

Según Manjarrés (2003), los costos de producción agrícola son todas aquellas erogaciones que realiza la empresa en el uso de los recursos o factores de producción con el fin de obtener un producto determinado. Así que, los costos totales de un cultivo comprende todos los desembolsos incurridos desde la preparación de suelos hasta la cosecha, esto es: Costo de producción agrícola es igual al gasto de explotación más inventario de cultivos en proceso menos inventario final de cultivo en proceso. Duque, H. (1993).

Gastos de explotación agrícola: Corresponde a cada uno de los desembolsos que se realizan durante el ciclo de producción.

Inventario de cultivos en proceso. Según Duque (1993), "Recoge el valor de los cultivos aún no recolectados, tomando por tal valor el costo invertido en el cultivo hasta la fecha" (p. 13). Puede ser el costo de los cultivos en proceso al comienzo del periodo (inventario inicial) o al final del mismo (inventario final).

\section{Clasificación de los Costos en las Empresas Agrícolas}

- Por fases de producción: preparación de suelos, siembra, labores culturales y cosecha.

- Por factores productivos: Mano de obra e insumos (semilla, abono, fertilizantes), maquinaria, tracción (Duque, 1982), citado por Manjarrés (2003, p. 76).

Algunos de los costos incurridos en cada una de las fases del proceso de producción de la papaya, son:

Preparación del suelo. Es un conjunto de actividades realizadas sobre el terreno donde se va a sembrar la semilla germinada, tales como, y arado, rastreo y surcado, las cuales generan costos. Entre los que tenemos:

Horas maquina por realización de las labores de labranza y surcado 
Siembra. La siembra de la semilla se realiza directamente en bolsas de polietileno negras de 20 x 14 cm, calibre 1. En la Provincia Mariscal Cáceres, los semilleros se establecen en enero-febrero para trasplantar en marzo-abril al inicio de las lluvias. Los mejores resultados se obtienen cuando el trasplante se hace seis semanas después de la germinación.

Al realizar el trasplante se eliminan las bolsas. Todas las plantas contenidas en una bolsa se siembran en un mismo hueco, de manera que el cuello de las raíces quede más alto que el nivel del suelo, pues ésta es la parte más susceptible a enfermedades favorecidas por la alta humedad. Se deben apartar algunas plantas para reemplazar las muertas.

La siembra de la semilla. Se incurre en algunos de los siguientes costos: Costo de la semilla, jornal por distribución de la semilla, jornal por tapado de la semilla, entre otros. Abonamiento. El cultivo de la papaya presenta un desarrollo rápido y constante acompañado de una floración precoz, continua y paralela al desarrollo de los frutos, por lo cual requiere de un suministro alto de agua y nutrientes durante todo el ciclo. Dentro de los factores que pueden modificar la producción agrícola están el uso de fertilizantes asociado a la irrigación como principal medio para aumentar la productividad del cultivo. Costo del abono Químico. Flete de traslado de abonos, Jornal por aplicación de abono químico

Labores culturales: Se refiere a determinadas prácticas, denominadas también labores de cultivo, que se realizan con el fin de mantener en buenas condiciones el suelo y favorecer el desarrollo de los cultivos. Las labores culturales, son: riego, control de malezas, aporque y fertilización y fumigaciones. En cada una de las cuales se generan costos, como los siguientes: Jornal para la realización del aporque, desahije y deshierbo; Costo mensual del sistema de riego.

Control Fitosanitario: Está definido dentro del cultivo al control de plagas y enfermedades que se puede presentar dentro de la producción con el manejo de fungicidas, insecticidas.

Jornal para la fumigación de fungicidas e insecticidas, costo de los agroquímicos (insecticidas, fungicidas)

Cosecha: La cosecha comienza a los 8 meses después del trasplante.

\section{Rentabilidad}


Rentabilidad es una noción que se aplica a toda acción económica en la que se movilizan unos medios, materiales, humanos y financieros con el fin de obtener unos resultados. En la literatura económica, aunque el término rentabilidad se utiliza de forma muy variada y son muchas las aproximaciones doctrinales que inciden en una u otra faceta de la misma, en sentido general se denomina rentabilidad a la medida del rendimiento que en un determinado periodo de tiempo producen los capitales utilizados en el mismo.

Rentabilidad en el análisis contable. La importancia del análisis de la rentabilidad viene determinada porque, aun partiendo de la multiplicidad de objetivos a que se enfrenta una empresa, basados unos en la rentabilidad o beneficio, otros en el crecimiento, la estabilidad e incluso en el servicio a la colectividad, en todo análisis empresarial el centro de la discusión tiende a situarse en la polaridad entre rentabilidad y seguridad o solvencia como variables fundamentales de toda actividad económica. Así, para Cuervo y Rivero (1986) la base del análisis económico-financiero se encuentra en la cuantificación del binomio rentabilidad-riesgo, que se presenta desde una triple funcionalidad: Análisis de la rentabilidad. Análisis de la solvencia, entendida como la capacidad de la empresa para satisfacer sus obligaciones financieras (devolución de principal y gastos financieros), consecuencia del endeudamiento, a su vencimiento. Análisis de la estructura financiera de la empresa con la finalidad de comprobar su adecuación para mantener un desarrollo estable de la misma.

Así, tenemos un primer nivel de análisis conocido como rentabilidad económica o del activo, en el que se relaciona un concepto de resultado conocido o previsto, antes de intereses, con la totalidad de los capitales económicos empleados en su obtención, sin tener en cuenta la financiación u origen de los mismos, por lo que representa, desde una perspectiva económica, el rendimiento de la inversión de la empresa.

$\mathrm{Y}$ un segundo nivel, la rentabilidad financiera, en el que se enfrenta un concepto de resultado conocido o previsto, después de intereses, con los fondos propios de la empresa, y que representa el rendimiento que corresponde a los mismos.

Por otro lado, los costos $\mathrm{ABC}$ es un método de análisis del costo que mide el costo y los resultados del proceso en que se relaciona las actividades y los objetos de costos. Analiza el costo de actividades basado en el uso de los recursos y lo asigna a los objetos de costos tales como productos o clientes basados en el uso de sus actividades. 
Este método analiza las actividades porque distingue dos verdades simples pero incuestionables: En primer lugar, no son los productos sino las actividades que causan los costos. En segundo lugar, son los productos los que consumen las actividades. Así es como se enlazan los costos con los productos por medio de las actividades, las cuales son causa de los primeros y son consumidos por los segundos.

La mayoría de los factores de costos son medidas del número de transacciones involucradas en una actividad particular por ello es que a los costos basados en actividades también se les denomina Costeo de Base de Transacciones.

\section{ESTRATEGIAS METODOLÓGICAS O MATERIALES Y MÉTODOS}

En la investigación se trazó una investigación correlacional transversal descriptiva, con el objeto de describir las características y relaciones de las variables estudiadas. La población estaba conformada por 50 productores del Cultivo de la papaya de la Provincia Mariscal Cáceres. 03 Productores del Distrito de Campanilla, 06 Productores del Distrito de Pajarillo, 39 Productores del Distrito de Juanjuí, 01 Productor del Distrito de Huicungo, 01 Productor del Distrito de Pachiza. El método utilizado fue Inductivo/deductivo, para efectuar generalizaciones de conceptos y comportamientos identificados, así como la particularización de comportamientos generales de la producción de la papaya y recomendar estrategias de rentabilidad. Las técnicas utilizadas han sido la observación y la encuesta, la entrevistas. Instrumentos el cuaderno de observación, cuestionario, guía de entrevista y cuaderno de campo. La tabulación se aplicó el programa Spps. Para la obtención de los datos.

\section{RESULTADOS Y DISCUSIÓN}

\section{RESULTADOS}

\section{Situación encontrada en los Costos de Producción del año 1}

Al 2007 inicio del proyecto Año 1 del costo de la producción del cultivo de la papaya en la preparación del terreno se tenía un total de s/410.00, con una participación del 34\% de aradura con tractor, el $49 \%$ corresponde al rastreo de tractor y el $17 \%$ del total de actividades al surqueo de tractor, en la que dicha actividad es esencial al inicio de este cultivo y realizadas en el mes de Octubre con pago de horas maquinas.

La estructura de los costos en la siembra desarrollada en los meses de Octubre a Noviembre es de s/ 780.00, donde el $38 \%$ corresponde al costo por Kg de semilla esencial para el inicio de este cultivo, el $21 \%$ del costo del manejo de vivero y el delineado de la 
siembra y el carguío manejan un total del $8 \%$, paseado es del 10\% y el $15 \%$ al trasplante del vivero al terreno del total de la siembra con jornales ejecutadas durante el proceso.

Es la fuente principal del cultivo de papaya para la fertilización de todo el proceso de producción se realizó en los meses de Diciembre para el abonamiento de la tierra estaba conformado por fertilizantes que se utiliza dentro del cultivo: como la Urea un $28 \%$, el $25 \%$ de Superfosfato triple, el 24\% de Cloruro de potasio, el 19\% de Granobol y el total de este proceso es de s/1004.00 la aplicación a realizarse es a mano que equivale a 2 jornales con un $4 \%$.del costo total del abonamiento.

En los deshierbo alcanza un $18 \%$ se mantiene en la misma magnitud, y los demás labores entre el $4 \%$ que implica el desahije y el aporque y el $21 \%$ del total al riego que se da en horas máquina de los labores culturales

El control fitosanitario con insecticidas alcanzo un 51\% y de fungicidas un $34 \%$ la aplicación de estos elementos se da con mochila fumigadora con jornales ejecutadas que equivale a un $15 \%$ del costo total de s/ 790.00

La cosecha de la papaya se da manualmente con jornales en un $62 \%$, de esta actividad así como; en la selección del fruto en un $15 \%$ y el carguío o acarreo corresponde a un $23 \%$ el flete no se realiza porque la comercialización se realiza en chacra, el total de esta actividad es de 260.00 nuevos soles.

El transporte de los insumos es el $100 \%$ para el total de la producción y de las jabas ó envases es un $0 \%$ por que corre a cuenta del comprador, el total de esta actividad es de 45.00 nuevos soles.

Los Costos Directos de producción del total de s/ 4385.00 nuevos soles, el imprevisto del total se consideró un 5\% que es de 219.25 nuevos soles y el costo financiero del $16 \%$ anual es de 701.6 nuevos soles.

Del total de las actividades constituidas para el año 01 es de s/ 4,385.00, el 9.35\% corresponde a la preparación del terreno por horas/ maquina, el $17.79 \%$ a la siembra manejo de vivero hasta el trasplante, el $22.90 \%$ abonamiento que implica el costo de abonos químicos y la aplicación de estos insumos se da con mochila fumigadora y con jornal ejecutado, el $24.99 \%$ a labores culturales $\mathrm{N}^{\circ}$ de Jornales, el $18.02 \%$ al control fitosanitario, $5.93 \%$ a la cosecha y el $1.03 \%$ a Gastos Varios donde se considera solo el transporte de insumos. 
Es el total de Costos Directos utilizados por un monto de 4385.00 nuevos soles y Costos Indirectos que corresponde a 920.85 nuevos soles dando un total de la Producción de 5305.85 nuevos soles, en el primer año de Instalación.

Se evidencia el total de costos de producción de papaya en el año 1 por 5305.85 nuevos soles que se obtiene por el rendimiento de la producción en una Ha de papaya * Kilogramo que es de 13440 kilogramos de papaya total por el precio unitario venta es de 0.30 céntimos de nuevos soles, dando un margen de 4032.00 nuevos soles el ingreso neto es ventas obtenidas menos costo de producción que equivale a -1273.00 nuevos soles generando una rentabilidad negativa $24.01 \%$ en el primer año.

\section{Gráfico $\mathbf{N}^{\circ} 1$}

\section{Costos por Actividad Directa}

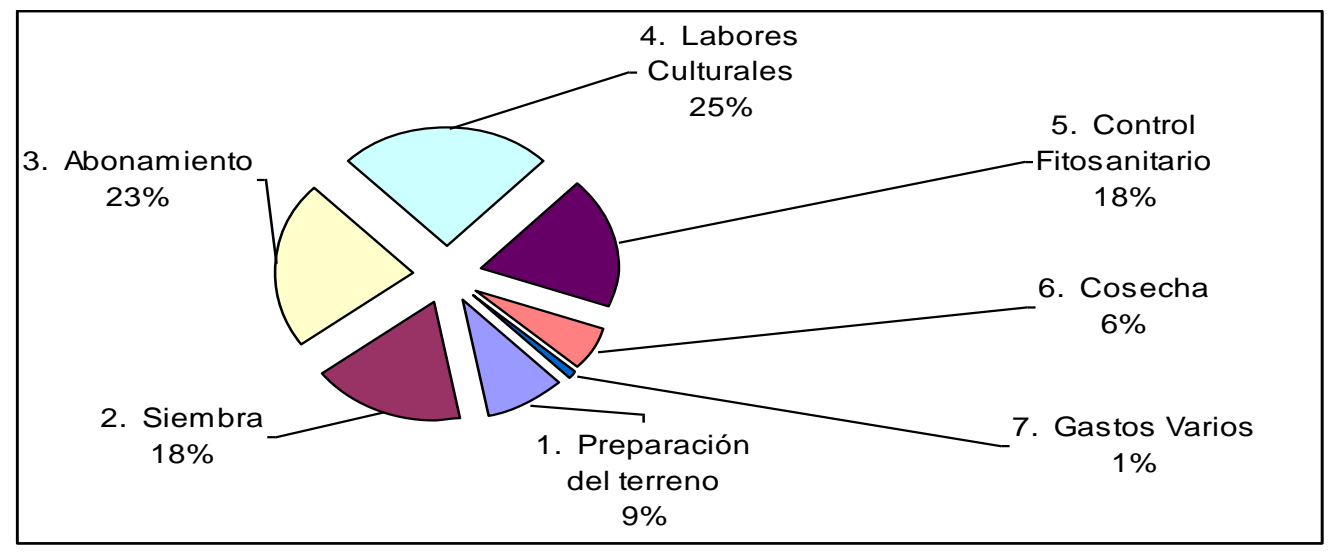

Fuente: Elaboración propia

\section{Situación de mantenimiento año 2}

En el segundo año de mantenimiento de la producción de la papaya se inicia con el abonamiento con un costo total de s/1362 donde el superfosfato es con un 32\%, urea con un $27 \%$ el cloruro de potasio con un $23 \%$ y el granobol con un $14 \%$, donde la aplicación corresponde al jornal durante el proceso y es del $4 \%$.

En el deshierbo de los diferentes procesos del cultivo alcanza un $22 \%$ de jornales ejecutadas, y en el riego del cultivo horas maquina es el 13\% del costo total de s/ 736.00 Los fungicidas para el control de enfermedades y plagas se consume del total el $51 \%$ de Ridomil y el Furadan el 34\% y la aplicación es con mochila con jornales que equivales el $15 \%$ con un total de 06 jornales del costo total de 790.00 nuevos soles.

La cosecha se realiza manualmente con jornales que representa un 62\%, el carguío, acarreo es el $25 \%$ en la selección de frutos, dentro de la cosecha es el $13 \%$ del total de s/ 
320.00 , en este proceso no se considera el flete a mercado porque la compra se realiza en la parcela del productor

El transporte de insumos es de s/ 600.00 que corresponde el 100\%, y el transporte de jabas y sacos corre a cuenta del comprador y dentro del costo es el $0 \%$.

En los costos directos se evidencia a partir del año 2 el abonamiento es fuente principal que equivale el $41 \%$ que equivale a 1362.00 nuevos soles, el control fitosanitario de la papaya es el $24.17 \%$ donde los labores culturales con horas jornales durante el mantenimiento es el $22.52 \%$, la cosecha es el $9.79 \%$ y el gasto varios que se considera el transporte de insumos es un $1.84 \%$ el total de costos directos en este año equivale a un monto de 3268.00 nuevos soles.

La participación de los costos directos é indirectos en el año 02 es de 3954.28 nuevos soles que equivale a mano de obra es del $28.83 \%$ de jornales ejecutada, los insumos en abonamiento y el control fitosanitario es del $52.30 \%$ que es un monto de 2068.00 nuevos soles, el transporte de insumos es el $1.52 \%$ en nuevos soles es de 60.00 y donde el imprevisto del costo ejecutado es de 163.40 nuevos soles que da un $4.13 \%$ y de costos financieros del $16 \%$ es de 522.88 nuevos soles que da el $13.22 \%$, de s/ 3268 de costos directos.

La participación de los costos directos del total de la producción de papaya es de 3268.00 nuevos soles y de los costos Indirectos es de 686.28 nuevos soles que equivale a 3954.28 nuevos soles en el año 2.

Durante el costo total de producción del cultivo de la papaya es de s/ 3954.28 durante el año 2 donde el rendimiento en una Hectárea es de 16500 Kilogramos, en donde el costo unitario por Kilogramo de fruta de papaya es s/ 0.24 promedio, y el precio unitario de venta es de s/ 0.30 donde el ingreso total del Valor Bruto Producido es de s/ 4950 ,este valor menos el costo del año 2, se obtiene una utilidad de s/995.72 obteniendo una rentabilidad o ganancia del $25.18 \%$.

\section{Gráfico $\mathbf{N}^{\circ} 2$}




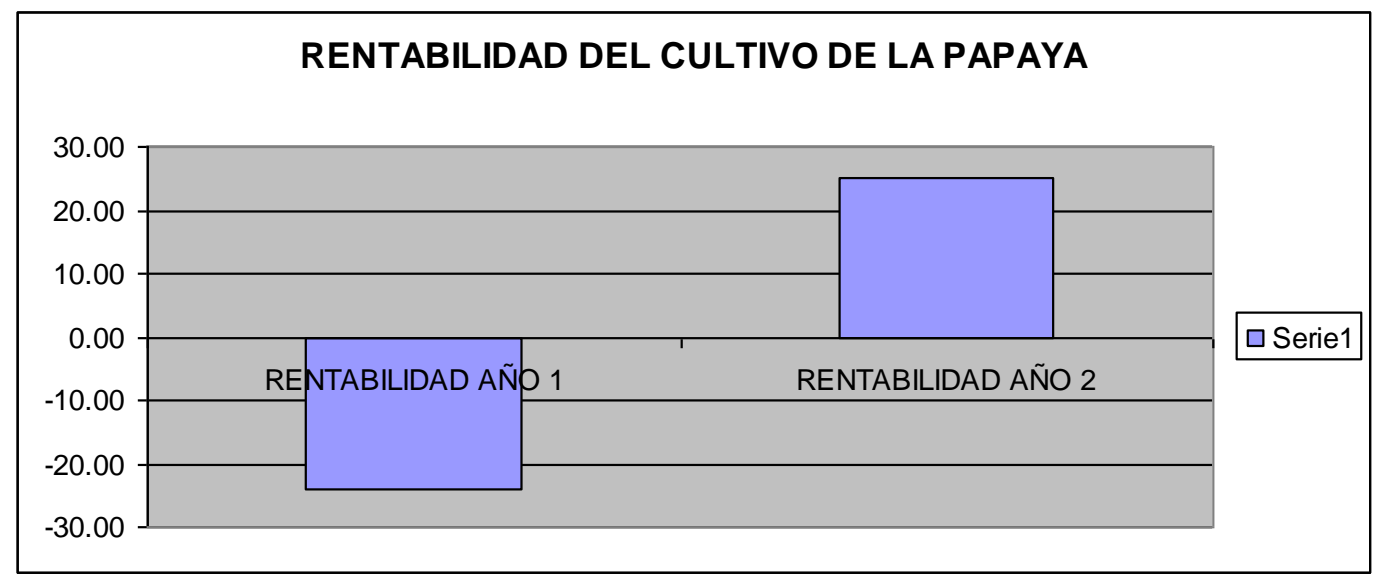

Fuente: Elaboración propia

Toda la producción tendrá como destino el mercado interno. El precio de venta en chacra por Kilogramo es de S/ 0.30 nuevos soles y donde la producción por año1 es de 13.44 Toneladas que equivale a 13440 kilogramos obteniendo una ganancia de S/ 4032 el segundo año se obtuvo 16500 kilogramos de producción de papaya que con las ventas obtenidas a 0.30 nuevos soles se obtuvo un monto de S/ 4950 nuevos soles en el segundo año.

\section{DISCUSIÓN}

Al 2007 inicio del proyecto Año 1 del costo de la producción del cultivo de la papaya en la preparación del terreno se tenía un total de s/410.00, con una participación del 34\% de aradura con tractor, el $49 \%$ corresponde al rastreo de tractor y el $17 \%$ del total de actividades al surqueo de tractor, en la que dicha actividad es esencial al inicio de este cultivo y realizadas en el mes de Octubre con pago de horas maquinas.

La estructura de los costos en la siembra desarrollada en los meses de Octubre a Noviembre es de s/ 780.00, donde el $38 \%$ corresponde al costo por Kg de semilla esencial para el inicio de este cultivo, el $21 \%$ del costo del manejo de vivero y el delineado de la siembra y el carguío manejan un total del $8 \%$, paseado es del $10 \%$ y el $15 \%$ al trasplante del vivero al terreno del total de la siembra con jornales ejecutadas durante el proceso.

Es la fuente principal del cultivo de papaya para la fertilización de todo el proceso de producción se realizó en los meses de Diciembre para el abonamiento de la tierra estaba conformado por fertilizantes que se utiliza dentro del cultivo: como la Urea un $28 \%$, el $25 \%$ de Superfosfato triple, el 24\% de Cloruro de potasio, el 19\% de Granobol y el total de este proceso es de s/1004.00 la aplicación a realizarse es a mano que equivale a 2 jornales con un $4 \%$.del costo total del abonamiento. 
En los deshierbo alcanza un $18 \%$ se mantiene en la misma magnitud, y los demás labores entre el $4 \%$ que implica el desahíje y el aporque y el $21 \%$ del total al riego que se da en horas máquina de los labores culturales

Durante el Periodo 2007 el control fitosanitario con insecticidas alcanzo un 51\% y de fungicidas un $34 \%$ la aplicación de estos elementos se da con mochila fumigadora con jornales ejecutadas que equivale a un $15 \%$ del costo total de s/ 790.00

La cosecha de la papaya se da manualmente con jornales en un $62 \%$, de esta actividad así como; en la selección del fruto en un $15 \%$ y el carguío o acarreo corresponde a un $23 \%$ el flete no se realiza porque la comercialización se realiza en chacra, el total de esta actividad es de 260.00 nuevos soles.

El transporte de los insumos es el $100 \%$ para el total de la producción y de las jabas ó envases es un $0 \%$ por que corre a cuenta del comprador, el total de esta actividad es de 45.00 nuevos soles.

Los Costos Directos de producción del total de s/ 4385.00 nuevos soles, el imprevisto del total se consideró un 5\% que es de 219.25 nuevos soles y el costo financiero del $16 \%$ anual es de 701.6 nuevos soles.

Del total de las actividades constituidas para el año 01 - 2007 es de s/ 4,385.00, el 9.35\% corresponde a la preparación del terreno por horas/ maquina, el $17.79 \%$ a la siembra manejo de vivero hasta el trasplante, el $22.90 \%$ abonamiento que implica el costo de abonos químicos y la aplicación de estos insumos se da con mochila fumigadora y con jornal ejecutado, el $24.99 \%$ a labores culturales $\mathrm{N}^{\circ}$ de Jornales, el $18.02 \%$ al control fitosanitario, $5.93 \%$ a la cosecha y el $1.03 \%$ a Gastos Varios donde se considera solo el transporte de insumos.

Es el total de Costos Directos utilizados por un monto de 4385.00 nuevos soles y Costos Indirectos que corresponde a 920.85 nuevos soles dando un total de la Producción de 5305.85 nuevos soles, en el primer año de Instalación.

Se evidencia el total de costos de producción de papaya en el año 1 por 5305.85 nuevos soles que se obtiene por el rendimiento de la producción en una Ha de papaya * Kilogramo que es de 13440 kilogramos de papaya total por el precio unitario venta es de 0.30 céntimos de nuevos soles, dando un margen de 4032.00 nuevos soles el ingreso neto es ventas obtenidas menos costo de producción que equivale a -1273.00nuevos soles generando una rentabilidad negativa $24.01 \%$ en el primer año. 
En el segundo año de mantenimiento de la producción de la papaya se inicia con el abonamiento con un costo total de s/1362 donde el superfosfato es con un 32\%, urea con un $27 \%$ el cloruro de potasio con un $23 \%$ y el granobol con un $14 \%$, donde la aplicación corresponde al jornal durante el proceso y es del $4 \%$.

En el deshierbo de los diferentes procesos del cultivo alcanza un 22\% de jornales ejecutadas, y en el riego del cultivo horas maquina es el 13\% del costo total de s/ 736.00 Los fungicidas para el control de enfermedades y plagas se consume del total el $51 \%$ de Ridomil y el Furadan el 34\% y la aplicación es con mochila con jornales que equivales el $15 \%$ con un total de 06 jornales del costo total de 790.00 nuevos soles.

La cosecha se realiza manualmente con jornales que representa un 62\%, el carguío, acarreo es el $25 \%$ en la selección de frutos, dentro de la cosecha es el $13 \%$ del total de s/ 320.00 , en este proceso no se considera el flete a mercado por que la compra se realiza en la parcela del productor

El transporte de insumos es de s/ 600.00 que corresponde el 100\%, y el transporte de jabas y sacos corre a cuenta del comprador y dentro del costo es el $0 \%$

En los costos directos se evidencia a partir del año 2 el abonamiento es fuente principal que equivale el $41 \%$ que equivale a 1362.00 nuevos soles, el control fitosanitario de la papaya es el $24.17 \%$ donde los labores culturales con horas jornales durante el mantenimiento es el $22.52 \%$, la cosecha es el $9.79 \%$ y el gasto varios que se considera el transporte de insumos es un $1.84 \%$ el total de costos directos en este año equivale a un monto de 3268.00 nuevos soles.

La participación de los costos directos é indirectos en el año 02 es de 3954.28 nuevos soles que equivale a mano de obra es del $28.83 \%$ de jornales ejecutada, los insumos en abonamiento y el control fitosanitario es del $52.30 \%$ que es un monto de 2068.00 nuevos soles, el transporte de insumos es el $1.52 \%$ en nuevos soles es de $60.00 \mathrm{y}$ donde el imprevisto del costo ejecutado es de 163.40 nuevos soles que da un $4.13 \%$ y de costos financieros del $16 \%$ es de 522.88 nuevos soles que da el $13.22 \%$, de s/ 3268 de costos directos.

La participación de los costos directos del total de la producción de papaya es de 3268.00 nuevos soles y de los costos Indirectos es de 686.28 nuevos soles que equivale a 3954.28 nuevos soles en el año 2 . 
Durante el costo total de producción del cultivo de la papaya es de s/ 3954.28 durante el año 2 donde el rendimiento en una Hectárea es de 16500 Kilogramos, en donde el costo unitario por Kilogramo de fruta de papaya es s/ 0.24 promedio, y el precio unitario de venta es de s/ 0.30 donde el ingreso total del Valor Bruto Producido es de s/ 4950 ,este valor menos el costo del año 2, se obtiene una utilidad de s/ 995.72 obteniendo una rentabilidad ó ganancia del $25.18 \%$

Toda la producción tendrá como destino el mercado interno. El precio de venta en chacra por Kilogramo es de S/ 0.30 nuevos soles y donde la producción por año1 es de 13.44 Toneladas que equivale a 13440 kilogramos obteniendo una ganancia de S/ 4032 el segundo año se obtuvo 16500 kilogramos de producción de papaya que con las ventas obtenidas a 0.30 nuevos soles se obtuvo un monto de S/ 4950 nuevos soles en el segundo año.

\section{CONCLUSIÓN O CONSIDERACIONES FINALES}

Se logró identificar los costos directos e indirectos de la producción del cultivo de la papaya como es en el año 1 instalación y en el año 2 mantenimiento.

Se determinó los costos directos de producción en la instalación y mantenimiento del cultivo de la papaya y los procesos dentro de la producción detallada (preparación de terreno, siembra, abonamiento, labores culturales, control fitosanitario, cosecha y gastos varios); de los costos indirectos que se obtuvo se consideró de imprevistos, el 5\% de los costos indirectos y el costo financiero del $16 \%$ anual.

Se logró establecer que el cultivo de la papaya en el año 1 su rentabilidad es negativa $24.01 \%$ y en el año 2 la rentabilidad es positiva logrando un $25.18 \%$ de rentabilidad,

Los análisis de rentabilidad elaborados en la estructura de costos de producción se llegó a la conclusión que el cultivo de la papaya no es una actividad rentable y no representa una alternativa importante para los agricultores a pesar que reúnen las características agro-ecológicas para producir papaya.

Los productores poseen mínima experiencia en el manejo del cultivo de la papaya por lo que requieren capacitación técnica y de organización, lo cual se solucionaría en la parcela de un productor, un módulo integrado que contemple los aspectos técnicos y sociales, que inciden en la producción y asesoría para mejorar el cultivo de costos en la producción e incrementar su rentabilidad en mejora de sus ingresos.

\section{REFERENCIAS BIBLIOGRAFICAS}


Adriazola, J.L. (1999). Tecnificación de los cultivos de papayo y cocona. Curso de Capacitación UNAS-IIAP. Tingo María, Perú

Aliaga, C. (1994). Manual de Matemática Financiera. Ed. Universidad del Pacifico.

Aliaga, C. (1991). Calculo Financiero - Operaciones, Activos. Ed. Editta

Armestar, N. y Guerra, L (1990). Ingeniería Económica. Lima: Códice Ediciones

Ayres, F. (1982). Matemática Financiera. México: Mc. Graw Hill

Avila, R. (1990). Introducción a la Metodología de la Investigación. CONCYTEC. Lima http://www.semillasdelcaribe.Como.mex/páginas/memorias/conferencias.thm Consulta del 03-08-036.

Bernal, C. (2000). Metodología de la Investigación para Administración y Economía. Editorial Prentice Hall. Bogotá.

Bernd, H. (2002). Exportar con Éxito-Ediciones Deusto. España.

BCRP (1995). Glosario de Términos Económicos. Lima

Bernstein, L. (1995): Análisis de estados financieros. Teoría, Aplicación e interpretación, Ed. Irwin, Madrid.

CAlzada, B. (1975). Cultivo del papayo. UNA La Molina. Lima, Perú.

Calzada B., J. (1983). Frutales nativos. Edit. Talleres gráficos UNALM. Lima, Perú.

CUERVO, A. (1994). Análisis y planificación financiera de la empresa, Ed. Civítas, Madrid.

Cuervo, A. y Rivero, P. (1986). El análisis económico - financiero de la empresa”, Revista Española de Financiación y Contabilidad. Nro 49, pp15-33.

Castillo, W. y Esteban, E. (2001). Normas Técnicas para redacción y presentación de Documentos Científicos. Editado por el CIUNAS - UNAS. Tingo María.

Cornejo, E. (1996). Comercio Internacional. Editorial San Marcos. Lima

DA COSTA, Joao (1992). Diccionario de Mercadeo y Publicidad. Editorial Panapo.

Caracas, Venezuela .274pag.

Escorche, V. (1990). Productividad y calidad. 1era. Edición. Editorial Nuevos Tiempos.

Esteo, F. (1998). Análisis contable de la rentabilidad empresarial, Centro de Estudios Financieros, Madrid.

Fernández, Jorge (1993). Dinero, Precios y Tipo de Cambio. CISP. Lima.

Gerald, A. (1994). Matemática Aplicada a Economía y Negocios. Editorial Gedisa. España. 
Gómez, L. (1992). Mejoramiento continuo de la calidad y productividad'2da. Edición.

Gonzales, J.(1995). Análisis de la empresa a través de su información económico financiera, Ed. Pirámide, Madrid.

Hernández, R.; Fernández, C. (2004). Metodología de la investigación científica. Edit. McGraw Hill. Bogotá, Colombia. 504 pp.

IPAE (1994). Como Administrar los Créditos y las Cobranzas. Lima Perú 201p.

Kotler, P. (1996). Dirección de Mercadotecnia. 8va edición. Prentice Hall.

Levitt, T. (1986). Comercialización Creativa. Compañía Editorial Continental. México.

Martínez, F. (1996) Análisis de estados Contables: Comentarios y Ejercicios, Ed.

Pirámide, Madrid.

Ocampo, E. (1991). Métodos de la Investigación Económica y Social, Editorial Horizonte. Lima

Ore, C. (1992). Estadística Descriptiva y Probabilidades. Princeliness E.I.R. Ltda. Lima

Porte, M. (1996). Estrategia Competitiva. Editorial Continental. México

Pares, A. (2001): Rentabilidad y endeudamiento ene. Análisis financiero y la planificación empresarial, Moneda y Crédito, Diciembre, nro.151, pp 3-20

Pride, W. (1997). Marketing: Concepto y Estrategias. 9na edición. Mc Graw - Hill.

Rivero, P. (1998). Análisis por ratios de los estados contables financieros (análisis externo), Ed. Civitas, Madrid.

Selling, T.I. y Stickney, C.P. (1990). Disaggregating the rate of return on common shareholders`equity: a new approach, Accounting Horizons, December.

Tawfick, L. (1994). Administración de la Producción $1^{\circ}$ Edit. Mc Graw- Hill, México D.F.

Urías, J. (1995). Análisis de Estados Financieros. Ed. Mc Graw - Hill, Madrid Yunus, M. (1997). Hacia un Mundo sin Pobreza. Editorial 